International Journal of Medical Anesthesiology 2019; 2(2): 44-47

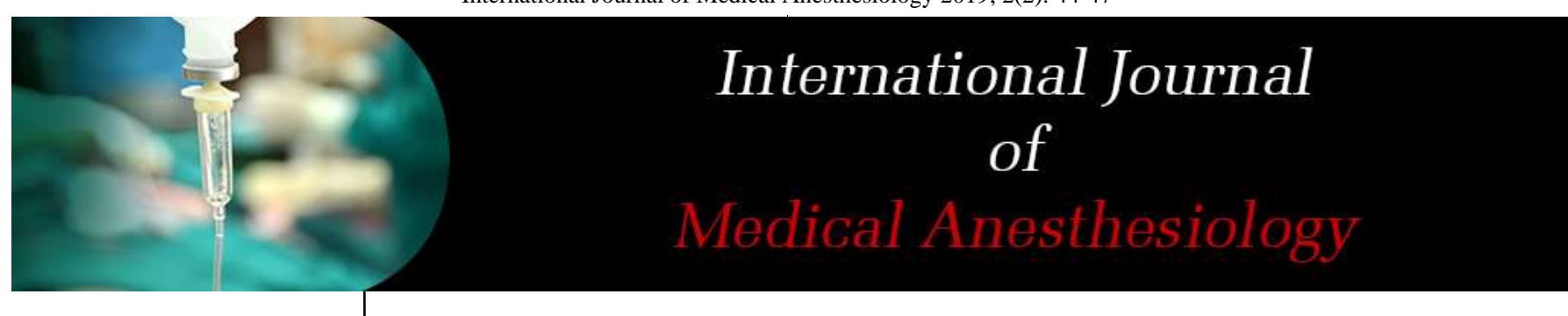

E-ISSN: 2664-3774

P-ISSN: 2664-3766

IJMA $2019 ; 2(2)$ : 44-47

Received: 12-05-2019

Accepted: 17-06-2019

Dr. Mahendra Singh Assistant Professor,

Department of Anaesthesia GCRG Medical College,

Lucknow, Uttar Pradesh, India

Dr. Dhiraj Prakash

Senior Consultant,

Department of General surgery

District Hospital, Ghazipur,

Uttar Pradesh, India
Corresponding Author: Dr. Dhiraj Prakash Senior Consultant, Department of General surgery District Hospital, Ghazipur, Uttar Pradesh, India

\section{Efficacy of oxycodone to facilitate general anaesthesia, haemodynamic changes and analgesia intra- and post- general surgery}

\author{
Dr. Mahendra Singh and Dr. Dhiraj Prakash
}

DOI: https://doi.org/10.33545/26643766.2019.v2.i2a.267

\begin{abstract}
Background: Haemodynamic changes and post-operative analgesia is vital to maintain during general anaesthesia to conduct mild to moderate surgery.

Objective: To evaluate the effect of oxycodone on physiologic changes following extubation and pain management after surgery.

Methods: As the sole opioid administered to induce and maintain general anesthesia, intravenous oxycodone was the sole opioid used in this prospective, observational, descriptive study. As well as recording the number of extubation times and adverse events, we observed all of these patients to see if oxycodone alone was sufficient to provide adequate intraoperative and postoperative analgesia.

Results: A total oxycodone dose of $0.35 \pm 0.06 \mathrm{mg} / \mathrm{kg}$ was used for induction and $32.4 \pm 3.21 \mathrm{mg} / \mathrm{kg}$ for maintenance of general anaesthesia. The dose was found to effectively maintain haemodynamic stability during surgery and good postoperative analgesia. A large number of patients took Oxycodone, resulting in deep sedation (five of the patients had sedation scores $\geq 4$ ) and respiratory depression, as well as long recovery times in the post-anaesthetic care unit. The extubation time $(18.5 \pm 2.6 \mathrm{~min})$ increased with increasing oxycodone doses.

Conclusion: For minor/moderate surgeries, oxycodone may be the only opioid used. Due to its deep sedation effect, care should be taken.
\end{abstract}

Keywords: General anaesthesia, general surgery, haemodynamic changes, analgesia, oxycodone

\section{Introduction}

After general anaesthesia, pain that is insufficiently controlled during the acute postoperative period can cause emergence agitation and interfere with the recovery process. Chronic pain may result from uncontrolled pain, which can delay discharge. In the acute postoperative phase, selecting the right analgesic is crucial. Postoperative pain can be effectively managed with opioid analgesics without severe side effects ${ }^{[1,2]}$. In comparison with other opioids, fentanyl starts acting quickly and has a shorter duration of action, so it is commonly prescribed for moderate to severe postoperative pain. Analgesic properties of oxycodone are longer lasting than those of fentanyl, and it provides a good analgesic effect for postoperative pain ${ }^{[3]}$. It is important for clinicians to understand a drug's pharmacological profile to choose an appropriate opioid dosage.

Oxycodone (14-hydroxy-7,8-dihydrocodeinone) is a semisynthetic opioid and exerts its biologic effect through the $\mu, \kappa$ and $\delta$ opioid receptors ${ }^{[4]}$. Taking advantage of oxycodone's rapid onset of action and its ability to last at least four hours after injection ${ }^{[5]}$, oxycodone intravenously has been studied and used for acute postoperative pain ${ }^{[6]}$, as well as for attenuating hemodynamic alterations in response to endotracheal intubation [6]. In comparison with morphine (lasts approximately 4 hours), oxycodone has a rapid onset time and a short duration of action ${ }^{[5]}$. When administered during induction, oxycodone may be sufficient to alleviate pain for 4 hours. Taking high doses of long-acting opioids during extubation can prolong it and increase adverse effects (such as depression, vomiting, and dizziness). There is no evidence, however, that it can be used alone for general anesthesia. Using oxycodone during general anaesthesia for induction and maintenance had no clinically significant effects on haemodynamics or respiratory function in one study ${ }^{[7]}$. There is insufficient information about the use of oxycodone alone for general anaesthesia, such as whether it provides ideal analgesia during general anesthesia and postoperatively, or whether it can pose adverse effects. Consequently, further research is needed to answer the following questions: can oxycodone be used as the sole opioid for general anesthesia? 
And when should anesthesia be extubated?

The purpose of this study was to evaluate the effect of oxycodone on physiologic changes following extubation and pain management after surgery.

\section{Materials and Methods Patients}

In this observational, descriptive study, all patients undergoing minor or moderate surgery (lasting less than 4 hours) required general anesthesia and tracheal intubation were included. The patients underwent general surgeries in this study, including conization of the cervix, laser treatment of laryngeal cancer, laparoscopic cholecystectomy, radical mastectomy, quadrant mastectomy, great saphenous vein high ligation and exfoliation, thyroidectomy, laparoscopic indirect hernia repair, laparoscopic hysterectomy, and laparoscopic pyeloureteroplasty. According to the American Society of Anesthesiologists (ASA), we included patients who were rated as class I or II. Patients were excluded if they: (1) had a body mass index $>30 \mathrm{~kg} / \mathrm{m} 2$ or $\leq 18.4 \mathrm{~kg} / \mathrm{m} 2$; (2) had a severe cardiovascular or respiratory disease; (3) had an expected difficult intubation; (4) had renal, liver, or haematological disorders; (5) had a high risk of aspiration or regurgitation; (6) were taking narcotics for chronic pain; or (7) had contraindications to oxycodone and flurbiprofen

\section{Treatments}

Patients did not receive any pre-medication before going into the operating room. In addition to pulse oximetry (SpO2), electrocardiography (ECG), and blood pressure (BP), and bispectral index (BIS) measurements, standard monitors were used. The baseline BP and heart rate (HR) were recorded. As described in our previous report, an IV bolus of oxycodone $(0.2-0.37 \mathrm{mg} / \mathrm{kg})$ was administered 5 minutes before propofol $(2 \mathrm{mg} / \mathrm{kg})$ and rocuronium $(0.8$ $\mathrm{mg} / \mathrm{kg}$ ) for induction, and tracheal intubation was performed approximately 2 minutes after induction. Using Dixon's upand-down method, the oxycodone dose for induction was altered to determine the ED50 of oxycodone ${ }^{[8]}$.

Continuous propofol infusions were used to maintain anaesthesia. A dose of cisatracurium was administered as needed. An additional dose of propofol was administered if the anaesthesiologist doubted the anaesthesia's effectiveness, and oxycodone $(0.03 \mathrm{mg} / \mathrm{kg})$ was administered when insufficient analgesia was determined.

When severe bradycardia (HR $>50$ beats/min) occurred, 0.5 $\mathrm{mg}$ of atropine was administered intravenously. Ephedrine $(6 \mathrm{mg})$ was used for hypotensive events and urapidil for the hypertensive events (both defined as BP reduced or increased, respectively by $30 \%$ than baseline). Just before the stitching began, propofol was stopped and postoperative care rooms were moved to the post-operatively awakened patients.

\begin{abstract}
Assessment
Haemodynamic parameters (BP and HR) were recorded at the following time points: T0, arrival in the operating room; $\mathrm{T} 1$, before intubation; T2, 3 minutes after intubation; T3, beginning of the operation; $\mathrm{T} 4$, end of the operation; and $\mathrm{T} 5$, extubation time. The extubation time (the time from the cessation of propofol infusion to extubation), the anaesthesia time (the time from injection of oxycodone to extubation), and the post-operative care unit time (total time in post-operative care unit) were also recorded.

The adverse events, sedation, and pain scores were assessed by blinded observers. Table 1 lists the Ramsey sedation score (RSS) used to assess sedation at admission to the postoperative care unit time.
\end{abstract}

Table 1: Description for Ramsey sedation score (RSS)

\begin{tabular}{|c|c|}
\hline Score & Description for patients \\
\hline 1 & The patient is anxious, agitated, or restless \\
\hline 2 & The patient is cooperative, oriented, and tranquil \\
\hline 3 & The patient responds to commands \\
\hline 4 & The patient gives a brisk response to a light glabellar tap or loud auditory stimulus \\
\hline 5 & The patient gives a sluggish response to a light glabellar tap or loud auditory stimulus \\
\hline 6 & The patient does not respond to stimuli \\
\hline
\end{tabular}

During the post-anaesthetic care unit and four, eight, and twenty-four hours after surgery, pain at rest was scored on a numerical scale from 0 to 10 , with 10 being the worst possible pain. Twenty-four hours after the operation, adverse events were recorded in the post-operative care room. An individual with respiratory depression must inhale oxygen through a mask and have a respiratory rate below 8 breaths per minute. Patient satisfaction was ranked by the patient as follows: 1, satisfactory; 2, neutral; and 3, unsatisfactory.

\section{Results}

Fifty-five patients were enrolled, but four patients were excluded. Three patients were excluded because oxycodone infusion caused hypertension, and one patient was excluded because of bleeding during the operation. Therefore, 51 patients were included in the final analyses. The average age of the patients was $51.4 \pm 10.2$ years, and 31 were men and 20 were women. The patients' demographic data and surgical profiles are shown in Table 1.

Table 1: Demographic characteristics of patients

\begin{tabular}{|c|c|}
\hline Characteristics & Patients $(\mathbf{n}=\mathbf{5 1})$ \\
\hline Age (Years) & $51.4 \pm 10.2$ \\
\hline Height $(\mathrm{cm})$ & $168.2 \pm 12.1$ \\
\hline Weight $(\mathrm{kg})$ & $59.8 \pm 9.9$ \\
\hline BMI $\left(\mathrm{kg} / \mathrm{m}^{2}\right)$ & $24.1 \pm 3.5$ \\
\hline Induction oxycodon dose $(\mathrm{mg} / \mathrm{kg})$ & $0.35 \pm 0.06$ \\
\hline Total maintenance oxycodon dose $(\mathrm{mg} / \mathrm{kg})$ & $32.4 \pm 3.21$ \\
\hline Operation time (min) & $72(35-225)$ \\
\hline Anaesthesia time (min) & $125(55-250)$ \\
\hline Extubation time (min) & $18.5 \pm 2.6$ \\
\hline Post-operative care unit (min) & $40(12-120)$ \\
\hline
\end{tabular}

During surgery, haemodynamic parameters, blood pressure and heart rate changed at different time points (Figure 1) but most remained within the clinically stable range (the changes were less than $20 \%$ of the baseline). 


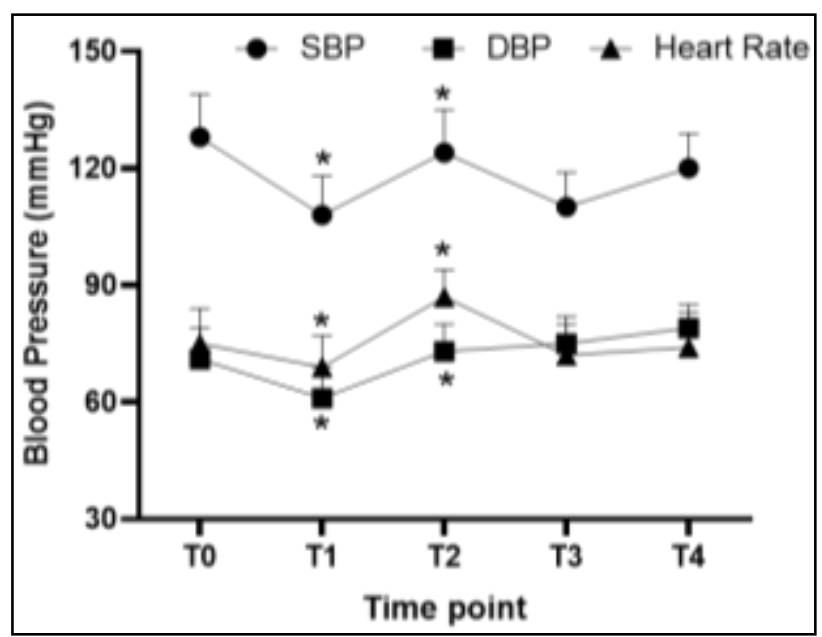

Fig 1: Blood pressure (Systolic and diastolic) and heart rate at different time points. The data are presented as the mean $\pm \mathrm{SD}$. *indicates $P<0.05$.

Oxycodone was effective for sedation and pain management in the post-anaesthesia care unit. Fifteen patients were in the deep sedation group (RSS $\geq 4$ ) (Figure 2). The postanaesthetic care unit time in the deep sedation group (RSS 24) was 58.5 (Range 30-110) minutes, which was longer than that in the less sedation group (RSS <4) $(34.8$ minutes (Range 16-72 minutes, $P<0.01$.

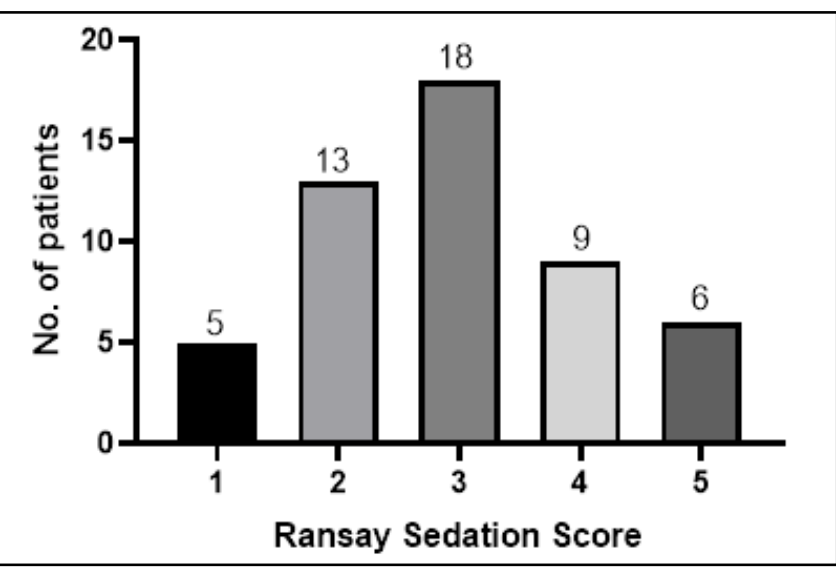

Fig 2: Ramsey sedation score (RSS) in the post-anaesthesia care unit. The data are presented as the number of cases with each score.

The NRS was $0(0-6)$ in the post-anaesthetic care unit. 8 patients felt pain and required additional tramadol in the post-anaesthetic care unit. NRS was $0(0-5), 1(0-5), 1$ (0$5)$ and $1(0-5)$ at 4, 8, 12 and 24 hours after the operation, respectively.

Adverse events that are associated with oxycodone (seen in the dispensatory), such as respiratory depression, vomiting, and dizziness, were recorded in the post-anaesthetic care unit and within 24 hours after surgery. Three patients had respiratory depression in the post-anaesthetic care unit due to deep sedation. Fourty-seven patients (96\%) had a satisfactory experience, two patients $(4 \%)$ had a neutral experience, and one patient $(2 \%)$ had an unsatisfactory experience due to severe vomiting.

\section{Discussion}

The use of oxycodone for general anaesthesia maintains haemodynamic stability and provides good intra- and postoperative pain management in minor/moderate surgeries.

There is widespread use of compound opioids (fentanyl and sufentanil to induce general anaesthesia, remifentanil to maintain it, and other analgesics for postoperative pain management) in current clinical practice for general anaesthesia. Performing calculations, converting, and determining the timing between the administration of multiple opioids requires an anaesthesiologist with extensive experience. Opioids such as oxycodone have short onset times and long durations of effect, making them suitable for induction and maintenance of anaesthesia or for management of postoperative pain ${ }^{[10-14]}$. Thus, we assumed that oxycodone would provide sufficient analgesia for the procedure and be easier for the anaesthesiologist to deliver. General surgery using oxycodone alone is not well studied. As oxycodone has a long duration of action, it may cause more adverse effects in short-term administration, especially during shorter operations, if it is used alone for general anesthesia. Therefore, we performed this observational, descriptive study of minor (such as conization of the cervix, laser treatment of laryngeal cancer, quadrant mastectomy) and moderate (such as laparoscopic hysterectomy, laparoscopic pyeloureteroplasty, thyroidectomy) surgeries with operation times ranging from $0.5 \mathrm{hr}$ to $4 \mathrm{hrs}$ to observe the feasibility of oxycodone as the sole opioid for general anaesthesia.

It is imperative for general anaesthesia not only during the surgery but as well that hemodynamic stability be maintained (blood pressure and heart rate changes less than $20 \%$ of baseline) operation, but also after the operation. Extubation and intubation, however, appeared to affect blood pressure and heart rate. The effect of oxycodone on hemodynamic instability associated with tracheal intubation has been reported ${ }^{[9]}$. Because the study was designed to determine the $\mathrm{ED}_{50}$ of IV oxycodone for anaesthetic induction using Dixon's up-and-down method, some inefficient oxycodone doses were used and caused the BP and HR to increase at T2 (3 minutes after intubation) and T3 (beginning of the operation). Anaesthesiologist added oxycodone $(0.03 \mathrm{mg} / \mathrm{kg})$ when insufficient analgesia was detected after infusing more propofol under BIS monitoring. Last, we determined oxycodone's feasibility and adverse effects with large total doses (approximately $0.35 \quad 0.05$ $\mathrm{mg} / \mathrm{kg}$ ). We found that oxycodone alone combined with propofol for general anaesthesia could maintain the haemodynamic stability during the operation (BP and HR changes were less than $20 \%$ of the baseline), which is a basic requirement of general anaesthesia. This outcome was similar to other reports ${ }^{[7]}$, which used smaller oxycodone doses $(0.075 \mathrm{mg} / \mathrm{kg}$ during induction and $0.05 \mathrm{mg} / \mathrm{kg}$ during maintenance), causing slight HR changes (previous intubation to recovery, 72.5 beats/minute vs. 78.3 beats/minute) without any obvious changes in BP. We used larger doses of oxycodone to better maintain haemodynamic stability during extubation.

The total dose of oxycodone that was used in this study was approximately $0.35 \pm 0.05 \mathrm{mg} / \mathrm{kg}$ (Table 1 ), which could be equivalent to the induction dose of fentanyl $(3-5 \mu \mathrm{g} / \mathrm{kg}$ ) because the potency ratio of fentanyl to oxycodone is approximately $1: 75$ to $1: 100$ (Hwang et al. 2014). As a consequence, an anaesthesiologist may find it easier to provide adequate analgesia with oxycodone for induction in minor/moderate surgeries. As a single opioid, oxycodone 
provided good analgesia postoperatively in this study. Only three patients received additional tramadol for pain relief in the post-operative care unit. In one case, the operation time was 225 minutes (approximately 4 hours), but the anaesthesia time was 250 minutes, which exceeded the action time of oxycodone, and an additional analgesic was required. Although the analgesic intensity decreased with time during the 24 hours after surgery (median NRS increased from 0 to 1 ), most of the patients had good pain management. During the first 24 hours after surgery, only one patient needed additional pain medication.

An oxycodone-only anaesthesia could result in excessive sedation if large doses of oxycodone are used. Fifteen patients had deep sedation ( $\mathrm{RSS} \geq 4$ ) in the pots-operative care unit, which led to longer post-operative care unit times (58.5, Range 30-110 minutes) and three cases of respiratory depression in the post-operative care unit. There were more adverse events in the oxycodone group than in the fentanyl group, which is associated with sedation ${ }^{[10,11]}$. Given the limitations of this study (fewer long operations), more study is needed to determine whether oxycodone reduces deep sedation during long operations (longer than 2 hours).

\section{Conclusion}

Pain management can be achieved using oxycodone as an opioid for general anaesthesia during minor/moderate surgery. Despite this, patients should take extra care because it may cause respiratory depression and prolonged hospitalization.

\section{References}

1. McQuay H. Opioids in pain management. Lancet 1999;353:2229-32.

2. Raeder J. Opioids in the treatment of postoperative pain:old drugs with new options? Expert Opin Pharmacother 2014;15:449-52.

3. Koch S, Ahlburg P, Spangsberg N, Brock B, Tønnesen E, Nikolajsen L. Oxycodone vs. fentanyl in the treatment of early post-operative pain after laparoscopic cholecystectomy:a randomised double-blind study. Acta Anaesthesiol Scand 2008;52:845-50.

4. King SJ, Reid C, Forbes K, Hanks G. A systematic review of oxycodone in the management of cancer pain. Palliat Med 2011;25(5):454-70.

5. Silvasti M, Rosenberg P, Seppala T, Svartling N, Pitkanen M. Comparison of analgesic efficacy of oxycodone and morphine in postoperative intravenous patient-controlled analgesia. Acta Anaesthesiol Scand 1998;42(5):576-80.

6. Lee YS, Baek CW, Kim DR, Kang H, Choi GJ, Park $\mathrm{YH}$, et al. Comparison of hemodynamic response to tracheal intubation and postoperative pain in patients undergoing closed reduction of nasal bone fracture under general anesthesia: a randomized controlled trial comparing fentanyl and oxycodone. BMC Anesthesiol. 2016;16(1):115.

7. Wirz S, Ellerkmann RK, Soehle M, et al. Oxycodone is safe and effective for general anesthesia. J Opioid Manag 2018;14:125-130.

8. Kang XH, Bao FP, Zhang HG, et al. Gender affects the median effective dose and $95 \%$ effective dose of oxycodone for blunting the hemodynamic response to tracheal intubation in narcotic-naive adult patients. Chin Med J (Engl) 2018;131:1958-1963.
9. Bao F, Xie Q, Zhang H, Zhu S, Kang X. Feasibility of using oxycodone as the sole opioid for induction and maintenance of general anaesthesia in minor/ moderate surgery: a prospective, observational, descriptive study. J Int Med Res 2020;48(10):300060520957500.

10. Hwang BY, Kwon JY, Kim E, et al. Oxycodone vs. fentanyl patient-controlled analgesia after laparoscopic cholecystectomy. Int J Med Sci 2014;11:658-662.

11. Koch S, Ahlburg P, Spangsberg N, et al. Oxycodone vs. fentanyl in the treatment of early post-operative pain after laparoscopic cholecystectomy: a randomised double-blind study. Acta Anaesthesiol Scand 2008;52:845-850. 